\title{
Development of a Non-invasive Pressure Sensor
}

\author{
Paul Szász', Vadim Migunov ${ }^{1}$ \\ ${ }_{1}^{1}$ ABB Corporate Research Center, Wallstadter Str. 59, 68526 Ladenburg, Germany \\ paul.szas@de.abb.com
}

\begin{abstract}
Summary:
A novel solution for measuring pressure non-invasively is presented, offering new perspectives for retrofittable monitoring and optimization applications. The clamp-on sensor is based on measurement of the circumferential deformation of pressurized pipes, using a compliant pre-stressed metal clamp with strain gauges as sensing elements. The design concept of this measurement system is presented, including the underlying functional mechanisms. Its achievable measurement performance and applicability limitations have been derived from long-term validation tests.
\end{abstract}

Keywords: non-invasive, pressure, sensor, strain gauge, clamp,

\section{Background, Motivation an Objective}

While non-invasive sensors are increasingly available for process variables like temperature or flow, pressure sensing is still persistently invasive, needing direct fluid contact. Filling this gap in non-invasive sensing is expected to offer significant benefit, e.g. regarding increased safety and hygiene due to zero leakage risk, or improved process control at low cost due to easy retrofit even in running processes.

In patent literature, solutions for this task are present since the 1970ies. Still no product has emerged over all these years, indicating major technical obstacles. One main limiter is the international pressure vessel design regulation, leading to highly stiff piping which is supposed to suffer minimum effects by internal pressure variations. While this is favorable for safety and longevity of the piping, it actually strongly limits the use of a process pipe as sensing element. Transforming the pressure into a measurable signal appears to require very elaborate and costly technologies. ABB has taken the chance to pioneer in this field and present a sensing concept which can overcome many of the so far known limitations at an acceptable cost level.

\section{Measurement Principle}

After analyzing the vast variety of physically conceivable measurement principles, strain gauge sensing of the pipe circumferential strain was selected as promising option. Strain gauge sensing offers not only sufficient resolution of the very small pipe deformations, but it is also widely available at moderate cost and well understood, so development could concentrate on the realization of a sensor system with sufficient robustness and reliability. Pipe circumferential strain is also favorable as measurand, as it is fully independent of the pressurizing fluid offering so a broad use range.

\section{Technical solution}

Direct strain gauge application to process pipes in the field is not practical for an industrial sensing device. So, a solution for transferring the pipe deformation to a sensor structure with a well-defined transfer function was developed. I.e. the deformation of this structure deviates in a predictable way from the deformation of the pipe. A flexible thin steel strip is wrapped around the pipe and pre-stressed to a degree which makes it fully compliant to the pipe surface (Fig.1).

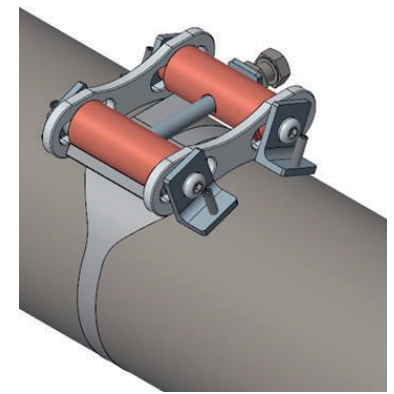

Fig. 1. Strain and pressure sensing clamp

As result, the strains on the clamp strip surface will always be almost identical to the pipe surface deformation. Having a small thermal mass and good contact to the pipe, the clamp also will have the same temperature, reducing temperature induced errors. Variations of the strain in the clamp over the circumference and especially due to the discontinuity at the tensioning mechanism can be accounted for using a transfer function determined by calibration, as they are constant properties of the clamp. 
The tensioning mechanism is an especially sensitive part, requiring high long-term stability and minimized influence on the actual transfer function. Its design included aspects of thermal and elastic tailoring, ease of handling and reliability.

\section{Temperature compensation}

Temperature induced signal variations are inherent to strain gauge sensing and are typically solved by using full Wheatstone bridge circuitry. However, on curved surfaces and especially on pressurized pipes there is no way to create such a self-compensating circuitry. Only a diagonal half bridge solution was applicable, measuring the circumferential strain on two locations of the clamp. Consequently, a solution was developed to quantify the thermal response of the clamp in a calibration step. The generated temperature dependent calibration values can subsequently be subtracted from the actual measured values, allowing the correction of the temperature induced error.

\section{Measurement of pressure step change}

The strain gauge clamp solution was thoroughly tested to provide information on the expectable measurement performance.

Pressure step changes, i.e. discrete pressure changes within defined time periods give a highly linear strain signal (Fig. 2).

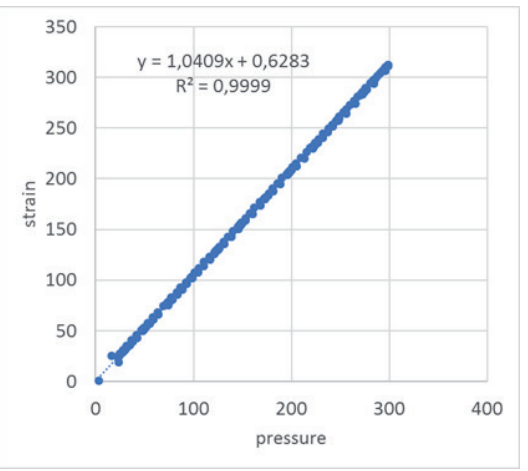

Fig. 2. Strain is a linear function of pressure

The strain signal is corrected by the known transfer function of the clamp to yield the actual pipe strain value. Knowing geometric and elastic properties of the pipe (described by according constants $\mathrm{k}_{\text {geom }}$ and kelast) any strain change $\Delta \varepsilon$ can be analytically transformed into a corresponding pressure change $\Delta p$ with an accuracy within $1-2 \%$ of the rated pressure of the considered pipe (eq. (1)).

$\Delta p=\Delta \varepsilon_{\text {pipe }} \times k_{\text {elast }} \times k_{\text {geom }}$

\section{Long term stability}

Experiments over several months showed that long term stability does not come up to the level of pressure step change measurement accuracy. Though drifts in the mechanical setup were excluded by design, two inherent stability issues are still prevalent. First, the accuracy of thermal calibration cannot fully exclude signal fluctuations in the order of up to $5 \%$ of the rated pressure of the pipe due to "stress augmented thermal activation" in the interfaces, leading to temperature gradient dependent reversible creep and friction phenomena. Additionally, long term slow drift of the zero signal was observed (inherent to glued strain gauges, especially on highly stressed components), leading to about $0.5 \%$ drift per month, which can be compensated by periodic zero reset.

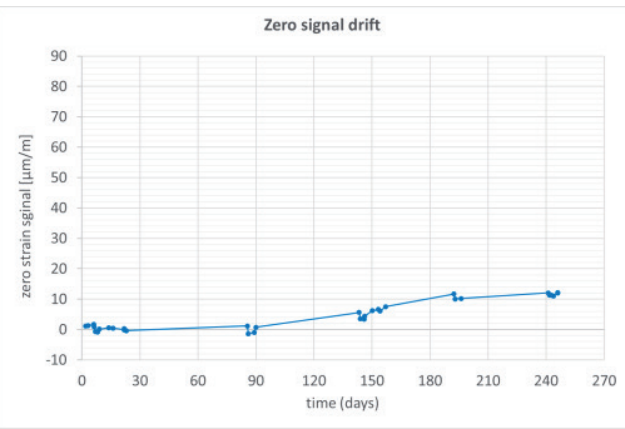

Fig. 3. Long term signal fluctuation and drift

\section{Applicability conclusions}

The developed clamp-on pressure sensor is scalable for pipe sizes exceeding DN50 based on the developed design rules. It is applicable for all pressure ratings exceeding 10 bars. For lower pressure piping the small deformations limit the achievable resolution and accuracy strongly.

It is important to note that the non-invasive pressure measurement always measures a pressure difference related to a defined starting point, usually the mounting state or an arbitrary zero setting. l.e. a one-point calibration (knowing the real pressure value at a given time) is essential to enable this measurement.

This non-invasive pressure sensor can detect and quantify short term pressure changes with an accuracy of $1-2 \%$, making it suited for monitoring of e.g. pump or valve functions, flow obstructions or leakage. Alternatively, for known pressure steps the system can recognize pipe wall thickness variations as may occur due to long-term corrosion.

Its long-term accuracy of 5 to $10 \%$ limits its use as absolute pressure sensor to applications with regular zero resetting possibility (e.g. batch processes) and small temperature variations, where the absolute accuracy can be $<2 \%$ of the pipe pressure rating over longer periods. 